\title{
Serum Creatinine and Blood Urea Nitrogen Levels in Patients with Coronary Artery Disease
}

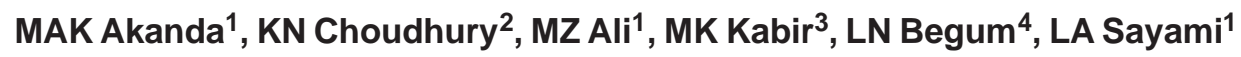 \\ ${ }^{1}$ National Institute of Cardiovascular Diseases, Dhaka, ${ }^{2}$ National Centre for Control of \\ Rheumatic fever and heart disease, Dhaka, ${ }^{3}$ Dhaka Medical College, Dhaka, ${ }^{4}$ Bangabandhu \\ Sheikh Mujib Medical University, Dhaka.
}

Key words:

Serum

creatinine, BUN, Coronary artery disease, Gensini scores.

\begin{abstract}
:
Background: Few studies have assessed the relation of Serum creatinine and serum blood urea nitrogen $(B U N)$ level with the severity of coronary artery disease (CAD). This study investigated the association between high uric acid BUN levels with the presence of Coronary artery disease.

Materials and Methods: This study was designed as an observational cohort study. The study was composed of 170 patients admitted at our institution due to symptoms related to CAD. Patients having angiographic evidence of stenosis in coronary artery were as case group and without stenosis control group. Patients with high serum creatinine were defined as serum creatinine concentration with in 80-105 $\mu \mathrm{mol} / \mathrm{L}$ and BUN level with in 10-20 $\mu \mathrm{mol} / \mathrm{L}$. The presence of $C A D$ has been defined as the Gensini score being $\geq 1$.

Results: Patients with or without CAD were similar in terms of age $(45.22 \pm 6.80$ years vs. $52.87 \pm 9.31$ years, $p<0.01)$ and significant age difference was found between patients. Male gender $(p<0.001)$ and smoking habit ( $p=0.003)$ were more frequent and statistically significant in patients with $C A D$. There was a statistically significant difference between the mean serum creatinine levels $(92.89 \pm 20.82 \mu \mathrm{mol} / \mathrm{L}$ vs $108.68 \pm 23.62$ $\mu \mathrm{mol} / \mathrm{L}$ respectively, $p<0.05)$ and serum blood urea nitrogen level $(10.59 \pm 6.15 \mu \mathrm{mol} / \mathrm{L}$ vs. $20.37 \pm 6.73$ $\mu \mathrm{mol} / \mathrm{L}$ respectively, $p<0.01)$ of patients with or without $C A D$. While looking at the correlation coefficient of Gensini score with different factors; $S$. creatinine, ejection fraction and BUN were significantly correlated at $<0.001$ and $<0.04$ and $<0.01$ level respectively. Increased serum creatinine levels were found to be independent risk factors for the presence of $C A D$ (for serum cretinine hazard ration 3.9, $p<0.001$ and in case BUN hazard ration 2.08, $p<0.001$ ).

Conclusion: In conclusion, a significant association has been found between serum creatinine \& BUN level and the presence of $C A D$. In addition to the evaluation of conventional risk factors in daily clinical practice, the measurement of serum creatinine and BUN level might provide significant prognostic benefits in terms of global cardiovascular risk and management of the patients.
\end{abstract}

(Cardiovasc. j. 2013; 5(2): 141-145)

\section{Introduction:}

It has been suggested that serum creatinine acts as a marker for generalized vascular disease. A number of studies indicate that elevated serum creatinine may be an independent predictor of all cause and of cardiovascular disease mortality. ${ }^{1-4}$ These studies have focused on specific groups, such as hypertensive individuals, ${ }^{5}$ and patients with coronary heart disease. The role of hypertension in determining the serum creatinine level in individuals has been examined in all of these studies but with no firm conclusion as to the primacy of blood pressure in determining the fatal outcome. The prognostic importance of serum creatinine in the incidence of coronary heart disease is uncertain.
Renal dysfunction has been associated with adverse cardiovascular outcomes. ${ }^{6}$ In these studied and others, renal function has routinely been assessed with an estimated creatinine clearance, serum creatinine or an estimated glomerular filtration rate derived from the serum creatinine. ${ }^{7}$

Serum blood urea nitrogen levels (BUN), however, may provide supplemental information in regard to renal function as renal proximal tubule cells may increase BUN reabsorption in the setting of increased neurohormonal activation. ${ }^{8}$ Accordingly, higher serum BUN has been associated with adverse outcomes in subjects with acute coronary syndrome. ${ }^{9}$

Address for Correspondence: Prof. Md. Abdul Kader Akanda, Department of Cardiology, National Institute of Cardiovascular Diseases, Dhaka, Bangladesh. 
Creatinine tests diagnose impaired renal function \& measure the amount of creatinine phosphate in the blood. BUN is an indirect \& rough measurement of renal \& live function measuring the amount of urea nitrogen in blood. BUN is directly related to the excretory function of the kidney.

This study investigated the association between serum creatinine and serum blood urea nitrogen levels with the presence of coronary artery disease.

\section{Materials and Methods}

The study was composed of 280 patients, between May 2011 and February 2012, were consecutively admitted at our institution due to symptoms related to CAD. All patients had elective coronary angiography at our centre. The patients were assessed a day prior to coronary angiography and a full clinical history was obtained. This study was designed as an observational prospective cohort study. Patients having angiographic evidence of stenosis in coronary artery or major branch segment in their epicardial coronary tree were as having CAD (Case group) and with out stenosis (Control group). Exclusion criteria were the first 4 weeks of acute coronary syndrome, previous percutaneous coronary intervention/stent implantation and/or previous coronary artery bypass grafting, presence of heart failure, alcohol consumption, patients with severely impaired renal function, neoplastic disease and chronic liver disease. Informed consent was obtained in accordance with the study protocol approved by the local ethical committee.

Coronary Angiography and Gensini Score: Coronary angiography was performed from the percutaneous femoral approach using standard angiographic techniques. The presence and severity of CAD was determined by clinical vessel score. All coronary angiograms were evaluated by two experienced cardiologists who were blinded to the laboratory results of the patients. The severity of the each lesion was assessed by quantitative coronary angiography.
The presence and total severity of CAD was assessed according to Gensini scoring system. In this system, angiographic stenosis between $1 \%$ and $25 \%$ is scored as 1 point, $26 \%$ and $50 \%$ is scored as 2 points, between $51 \%$ and $75 \%$ is scored as 4 points, between $76 \%$ and $90 \%$ is scored as 8 points, between $91 \%$ and $99 \%$ is scored as 16 points and total occlusion is scored as 32 points. These scores are multiplied by the coefficient defined for each coronary artery and segment, and the results are then added. The presence of CAD has been defined as the Gensini score being $\geq 1$.

\section{Definitions of CAD Risk factors:}

Analyzed risk factors of CAD were age, male sex, smoking, hyperlipidemia, diabetes, hypertension, family history of CAD and hyperuricemia. Hyperlipidemia was defined as plasma total cholesterol level $\geq 200 \mathrm{mg} / \mathrm{dl}$, LDLcholesterol level $\geq 130 \mathrm{mg} / \mathrm{dl}$, triglyceride level $\geq 150 \mathrm{mg} / \mathrm{dl}$ and HDL-cholesterol level $\leq 40 \mathrm{mg} / \mathrm{dl}$ or being on lipid lowering drugs at the time of the study. Patients were considered to have hypertension if they had received such a diagnosis with arterial pressure of more than $140 / 90 \mathrm{mmHg}$ or were being treated with antihypertensive medications. Patients were considered to have diabetes if they were taking insulin or oral hypoglycemic agents. Patients with a lack of awareness of their past history of diabetes were defined as a fasting blood glucose $\geq 120 \mathrm{mg} / \mathrm{dl}$. Patients with High creatinine concentration were defined as $\geq 1.2 \mathrm{mg} / \mathrm{dl}$ or $\geq 105$ $\mu \mathrm{mol} / \mathrm{L}$. Normal range are $0.9-1.2 \mathrm{mg} / \mathrm{dl}$ or 80 $105 \mu \mathrm{mol} / \mathrm{L}$. Normal BUN levels of BUN are between $10-20 \mathrm{mg} / \mathrm{dl}$.

\section{Results:}

Patients were allocated into two groups in terms of presence of CAD. Patients with Gensini scores $\geq 1$ were defined as the group with CAD and 134 patients were allocated in this group. Gensini score of 0 were classified as the group without CAD and 36 patients were included in this group. Demographic characteristics, the distribution of cardiovascular risk factors, Serum creatinine and serum blood urea nitrogen are presented in Table I. 
Table-I

Univariate analysis of coronary artery involvement with demographic and clinical profile

\begin{tabular}{lccc}
\hline Univariate analysis* & \multicolumn{2}{c}{ Coronary Artery involvement } & P value \\
& No $(36)$ & Yes $(134)$ & \\
\hline Age & $45.22 \pm 6.80$ & $52.87 \pm 9.31$ & 0.01 \\
S. creatinine & $92.89 \pm 20.82$ & $108.68 \pm 23.62$ & 0.05 \\
S. Cholesterol & $162.00 \pm 44.42$ & $175.81 \pm 52.02$ & 0.62 \\
HDL & $37.22 \pm 8.81$ & $36.26 \pm 7.86$ & 0.77 \\
LDL & $96.89 \pm 34.90$ & $102.07 \pm 42.22$ & 0.99 \\
TG & $173.89 \pm 109.45$ & $202.41 \pm 116.39$ & 0.04 \\
Ejection fraction & $61.78 \pm 7.23$ & $55.06 \pm 9.26$ & 0.02 \\
SGPT & $31.44 \pm 13.52$ & $37.81 \pm 23.70$ & 0.47 \\
BUN & $10.59 \pm 6.15$ & $20.37 \pm 6.73$ & 0.01 \\
Sex & & & \\
Male & $20(55.6)$ & $14(10.4)$ & $<0.001$ \\
Female & $16(44.4)$ & $120(89.6)$ & \\
Smoking & & & 0.003 \\
No & $28(77.8)$ & $40(29.9)$ & \\
Yes & $8(22.2)$ & $94(70.1)$ &
\end{tabular}

Patients with or without CAD were similar in terms of age $(45.22 \pm 6.80$ years vs. $52.87 \pm 9.31$ years, $\mathrm{p}<0.01$ ) and significant age difference was found between patients. Gender difference was statistically significant $(p<0.001)$. Smoking habit was significantly more frequent in patients with $\mathrm{CAD}(\mathrm{p}=0.003)$ and statistically significant.

There was a statistically significant difference between the mean serum creatinine levels of patients with or without CAD $(92.89 \pm 20.82 \mu \mathrm{mol} /$ $\mathrm{L}$ vs $108.68 \pm 23.62 \mu \mathrm{mol} / \mathrm{L}$ respectively $(\mathrm{p}<0.05)$. There was a statistically significant difference between serum blood urea nitrogen of patients with or without CAD $(10.59 \pm 6.15 \mathrm{mg} / \mathrm{dl}$ vs. $20.37 \pm 6.73$ respectively, $\mathrm{p}<0.01$.

There was no significant difference between patients with or without CAD in terms of the total cholesterol, LDL-c, HDL-c and family history. The $\mathrm{p}$ values for continuous variables are taken from Mann-Whitney U test.

The univariate analysis of the variables are shown in Table I showing age, serum creatinine, ejection fraction, lipid profile and BUN among continuous variables, while age, serum creatinine, ejection fraction, TG and BUN, are significantly associated with coronary artery involvement.
While looking at the correlation coefficient of Gensini score with different factors, S. creatinine, ejection fraction and BUN were significantly correlated at $<0.001$ and $<0.04$ and $<0.01$ level respectively. Increased serum creatinine levels were found to be independent risk factors for the presence of CAD (for serum creatinine hazard ration $3.9, \mathrm{p}<0.001$ and in case BUN hazard ration 2.08, p<0.001).

Table-II

Correlation of Gensini score and other continuous variables

\begin{tabular}{lcc}
\hline Correlation & Correlation coefficient & $\mathrm{p}$ value \\
\hline Age & 0.09 & 0.31 \\
S. Creatinine & 0.34 & $<0.001$ \\
Total Cholesterol & 0.01 & 0.88 \\
HDL & -0.04 & 0.66 \\
LDL & -0.09 & 0.27 \\
Triglyceride & -0.05 & 0.56 \\
Ejection fraction & -0.18 & 0.04 \\
SGPT & -0.04 & 0.63 \\
BUN & -0.17 & 0.01 \\
\hline
\end{tabular}

After getting the significant variables from univariate analysis, they were taken into the model of linear regression (Table II) to see their overall effects on Gensini score to learn that there is a significant overall effect $(\mathrm{F}=5.43$, 
$\mathrm{p}<0.001$ ) of these factors together on Gensini score. But the coefficients show that the significant effect was due to the impact of S. creatinine. So smoking habit was removed from the model as being non-significant and adjusting the new model with age and sex, S. creatinine, BUN and ejection fraction were significant variables $(\mathrm{F}=6.26, \mathrm{p}<0.001)$ to show their impacts on $\mathrm{CAD}$. The result of this regression analysis is shown below in Table (III).

\section{Table-III}

Final regression model with the significant variables on Gensini score, adjusted for age and sex

\begin{tabular}{lcc}
\hline Regression factors & $\mathrm{t}$ & $\mathrm{p}$ value \\
\hline Age & 0.27 & 0.79 \\
Sex & 1.4 & 0.16 \\
Serum creatinine & 3.91 & 0.001 \\
Ejection fraction & -1.98 & 0.04 \\
BUN & 2.08 & 0.001 \\
\hline
\end{tabular}

\section{Discussion:}

We had find that elevated serum creatinine and BUN on admission was associated with an increased burden of CAD on cardiac catheterization during index hospitalization in patients who presented with symptoms of unstable angina and without known cardiovascular disease. To the best of our knowledge, this study is the first to demonstrate this association. Furthermore, each $1 \mathrm{mg} / \mathrm{dl}$ increase in BUN was associated with an increased burden of CAD.

Blood urea nitrogen may have pro-athersclerotic effects, as uremia has been associated with an increased burden of oxidative stress. ${ }^{10}$ BUN may also both inhibit nitric oxide synthesis and promote macrophage proliferation. ${ }^{11}$ Specially, in vivo studies demonstrate that increasing levels of urea inhibt nitric oxide synthesis in mouse macrophages with concurrent macrophage proliferation. Furthermore, uremia accelerates atherosclerosis in Apolipoprotein E-deficient mice.

Other studies indicate that uremia induces the expression of osteoblast differentiation factor Cbfa1 in the intima and media of arteries, which may lead to vascular calcification. ${ }^{12}$ Elevated creatinine and BUN may also serve as a marker of an activated sympathetic nervous system and/ or an up regulated rennin-angiotensin system, reported promotes of atherosclerosis. ${ }^{13}$ Activation of these neurohormonal systems has been associated with increased BUN reabsorption in the renal tubules.

We observed this association between increased creatinine and BUN with CAD while correcting for creatinine clearance. Concurrent reports show that increasing BUN predicts poor outcome in subjects with acute coronary syndromes despite normal or mildly reduced glomerular filtration rates. Consequently, an elevated serum creatinine and BUN may represent an independent marker of renal dysfunction, which would further support the well established association between renal disease and CAD. ${ }^{14}$ However, additional study is required to evaluate this point.

\section{Limitations:}

Our study was observational in nature, which possibly restricted us in identifying and analyzing all potential confounding factors. The serum creatinine and BUN levels were a single measurement are not necessarily a reflection of one's chronic levels. Serum creatinine and BUN elevation may occur for reasons other than neurohormonal up-regulation and/or the presence of renal dysfunction. Analysis was based on a single measurement of creatinine and BUN the changes in levels over time are likely to occur. The main limitation that needs to be acknowledged regarding this study is the possible and unknown effects of the ongoing medications including diuretics.

\section{Conclusion:}

In conclusion, a significant association has been found between serum creatinine and BUN level with the presence of $\mathrm{CAD}$. In addition to the evaluation of conventional risk factors in daily clinical practice, the measurement of creatinine and BUN level might provide significant prognostic benefits in terms of global cardiovascular risk and management of the patients. Further study is warranted to explore this association.

Conflict of Interest - None. 


\section{References:}

1. Anvekar.N.S, McMurray JJ et al. Relation between renal dysfunction and cardiovascular outcomes after muocardial infarction. J Interna Med 1991; 351(13):1285-1295.

2. Nauer JH Brooks CS, Butch RN Renal function studies in man with advanced renal insufficiency. Am J kidney Dis 1982; 11: 30-35.

3. Cockcroft DW, Gault MH. Prediction of creatinine clearance from serum creatinine. Nephron 1976;16:31-41.

4. Doolan PD, Alpen EL et al. A clinical appraisal of the plasma concentration and endogenous clearance of creatinine. Am J Med 1962; 32:65-79 .

5. Dosseter JB Creatinine versus uremia. Ann Intern Med 1966; 65: 1287-1299.

6. McCullough PA, Interface between heart disease and renal dtsfunction from association. ACC Curr J Rev 2003; 12: 20-24.

7. Suwaidi JA. Reddan D.N et al. Prognostic implications of abnormalities in renal function in patients with acute coronary syndromes. J Fam Pract 1993; 36: 497-503.

8. LeShilpak MG, Heldenreich PA, et al. Association in renal insufficiency with treatment and outcomes after myocardial infarction in elderly patients. Ann Intern Med 1988; 18: 555-562.

9. Levey AS, Bosch JP et al. A more accurate method to estimate glomerular filtration rate from serum creatinine: A new prediction equation. Ann Intern Med 1999; 130: 461-470.

10. Himmelfarb J. Stenvinkel et al The elephant in uremia: Oxidative stress as a unifying concept of cardiovascular disease in uremia. Kidney Int J 1992; 62: 2524-2538.

11. Conte G, Canton A, et al. Renal handling of urea in subjects with persistent azotemia and noemal renal function. Kidney Int $J$ 1998; 32:721-727.

12. Moe S, Duan D et al. Uremia induces the osteoblast differentiation factor Cbfa1 in human blood vessels. Kidney Int $J$ 2003; 63: 1003-1011.

13. Kirtane AJ, Leder DM et al. Serum blood urea nitrogen as an independent marker of subsequent mortality among patients with acute coronary syndromes and normal to mildly reduced glomerular filtration rates. $J$ Am Coll Cardiol 2005; 45:1781-1786.

14. Feig Anvekar NS, McMurray JJ et al. Relation between renal dysfunction and cardiovascular outcomes after myocardial infarction. N Engl J Med 2004; 351(13):1285-1295. 